\title{
Breeding value of partial waxy wheat samples in Tatarstan
}

Askhadullin D-1 F.*, Askhadullin D-r F., Vasilova N.Z.

Tatar Scientific Research Institute of Agriculture, KSC RAS, Kazan, Russia

*e-mail: tatnii-rape@mail.ru

Non-functional alleles of $w x$ genes affect the violation of synthesis and changes in the localization of amylose in cereal starch. Wheat samples carrying non-functional alleles of $w x$ genes at one or two loci are called partial waxy wheat. From crossing the winter wheat variety Starshina (have non-functional allele $W x-A 1 b$ ) and the line of spring wheat O-192-03-5 (have non-functional allele $W x-B 1 b$ ) we obtained two promising lines of partial waxy wheat, which combines non-functional alleles $W x-A 1 b$ and $W x-B 1 b$. These are the K-243-13 Wx-2 and K-243-13Wx-6 lines. Test these lines was conducted in the Tatar Research Institute of Agriculture in 2017-2018. Tatar RIA is located in the northern part of the Middle Volga region of Russia. The average yield of the line K-243-13Wx-6 was $276 \mathrm{~g} / \mathrm{m}^{2}$, which is much less than the standard variety Yoldyz $-550 \mathrm{~g} / \mathrm{m}^{2}$. The line $\mathrm{K}-243-13 \mathrm{Wx}-2$ has an average yield of $534 \mathrm{~g} / \mathrm{m}^{2}$. The average weight of 1000 grains at the line K-243-13Wx-2 was $49.6 \mathrm{~g}$. At the line K-243-13Wx-2 degree of lesion of leaf rust was $0-15 \%$, the degree of damage of stem rust was $15 \%$. This line is susceptible to powdery mildew, its resistance is 3 points ( 9 points - maximum). Line K-243-13Wx-6 is susceptible to leaf rust, the degree of damage was $15-50 \%$. Line K-243-13Wx-6 is susceptible to stem rust, the degree of damage was 30-70\%. Resistance to powdery mildew in this line in epiphytotic 2017 was 4 points. At the line K-243-13Wx-2 date of earing before on 1 day, than at the line O-192-03-5. At the line K-243-13Wx-6 date of earing occurred simultaneously with the line O-192-03-5. According to the analysis of the harvested grain in 2018 year, the lines K-243-13Wx-2 and K-243-13Wx-6 have a high protein content in the grain of 14.9 and $14.5 \%$, respectively, and have a high gluten content in the grain of 30.8 and $31.7 \%$, respectively. Thus, the evaluation of agronomically valuable properties of the obtained samples of partial Waxy wheat indicates the prospects of their use as a starting material for the production varieties of spring wheat with a modified composition of grain starch. 